KYUNGPOOK Math. J. 49(2009), 451-456

\title{
Some Results of QF Rings
}

DONG JuN

The Basic Courses Department of Lanzhou Polytechnic College, Lanzhou 730050, China

e-mail : dongj@lzptc.edu.cn

Abstract. Let $R$ be a ring. We give some new characterizations of $Q F$ under the special annihilators condition. Some known results are obtained as corollaries.

\section{Introduction}

Throughout this paper, all rings are associative with identity and all modules are unitary. As usual, we use $J(R), Z\left({ }_{R} R\right), Z\left(R_{R}\right), \operatorname{Soc}\left({ }_{R} R\right)$ and $\operatorname{Soc}\left(R_{R}\right)$ (briefly $\left.J, Z_{l}, Z_{r}, S_{l}, S_{r}\right)$ to indicate the Jacobson radical, the left singular ideal, right singular ideal, and the left socle and right socle of the ring $R$, respectively. The left and right annihilators of a subset $X$ of $R$ are denoted by $l(X)$ and $r(X)$, respectively. We use $N \leq_{e} M$ to indicate that $N$ is an essential submodule of $M$.

Recall that a ring $R$ called right mininjective[1] if every right $R$-homomorphic from any minimal right ideal of $R$ into $R$ is given by left multiplication by an element of $R$. A ring $R$ is said to be right simple injective if every homomorphism from a right ideal of $R$ to $R$ with simple image can be given by left multiplication by an element of $R$ [2].It is clear that right simple injective rings imply right mininjective rings.

A ring $R$ is called quasi-Frobenius, briefly $Q F$, if $R$ is two-sided Artinian and two-sided self-injective ring, or equivalently, if $R$ has the $A C C$ on right or left annihilators and is right or left self-injective. The class of quasi-Frobenius rings is one of the most important classes of rings, which was introduced as a generalization of group algebras of a finite group over a field(see[3]). There are three open conjectures on $Q F$ rings, which have attracted many people to work on them. One of the three conjectures is the Faith-Menal conjecture. Faith's conjecture is an outstanding problem about quasi-Frobenius rings, which has been intensively investigated by various of authors(see[4] and [5] for more). There is a great deal of research devoted to improve this result by weakening the Artinian condition or the injectivity or both. Nicholson and Yousif[1] proved that any right and left mininjective ring, right Artinian ring is $Q F$. In [6], Nicholson and Yousif extended the condition from Artinian ring to a semilocal ring with $A C C$ on right annihilators

Received November 27, 2006; received in revised form July 13, 2007; accepted August 14, 2009.

2000 Mathematics Subject Classification: 16D50; 16L60.

Key words and phrases: P-injective ring, mininjective ring,special annihilators, QF-ring. 
such that $S_{r} \leq_{e} R_{R}$.

Because of these results, it is natural to ask whether these results are also correct when weaker condition of Noetherian ring or $A C C$ on left annihilators. For this purpose, we will consider the following condition for a given ring $R$ :

(*) The ascending chain $r\left(S_{1}\right) \subseteq r\left(S_{2} S_{1}\right) \subseteq \cdots$ terminates for any sequence $\left\{S_{1}, S_{2}, \cdots\right\} \subseteq R$.

Obviously, Artinian rings, Noetherian rings and ring having $A C C$ on left annihilators satisfy $(*)$.

First we show that if $R$ is a right mininjective ring and $R$ satisfies $(*)$ such that $S_{r} \leq_{e} R_{R}$, then $R$ is semiprimary . From this, we have (1) if $R$ is a left and right mininjective ring with $J^{2}=0$ and $R$ satisfies $(*)$ such that $S_{r} \leq_{e} R_{R}$, then $R$ is quasi-Frobenius; (2) if $R$ is a right simple injective ring with $J^{2}=0$ and $R$ satisfies (*) such that $S_{r} \leq_{e} R_{R}$, then $R$ is quasi-Frobenius. General background material can be found in Anderson and Fuller[7].

\section{Ring of satisfies condition $(*)$}

We start with the following lemma.

Lemma 2.1. Let $R$ be a ring satisfies (*). Then the following hold:

(1) For any sequence $a_{1}, a_{2}, \cdots \in R$, there is a positive integer $n$ such that $r\left(a_{n+1}\right) \cap$ $a_{n} \cdots a_{1} R=0$.

(2) If a right ideal $K$ of $R$ is right $T$-nilpotent, then $K$ is nilpotent.

(3) $Z_{r}$ is right nilpotent.

Proof. (1) By hypothesis, there is a positive integer $n$ such that $r\left(a_{n+1} \cdots a_{1}\right)=$ $r\left(a_{n} \cdots a_{1}\right)=\cdots$. For any $t \in r\left(a_{n+1}\right) \cap a_{n} \cdots a_{1} R$, then there is $r \in R$ such that $t=a_{n} \cdots a_{1} r$ and $r\left(a_{n+1}\right) t=0$ and so $a_{n+1} \cdots a_{1} r=0$,i.e., $r \in r\left(a_{n+1} \cdots a_{1}\right)=$ $r\left(a_{n} \cdots a_{1}\right)$. Thus $t=0$ and so $r\left(a_{n+1}\right) \cap a_{n} \cdots a_{1} R=0$.

(2) Assume that $K$ is not nilpotent, then $K^{n} \neq 0$ for any positive integer $n$. By hypotheses there exists a natraul number $m$ such that $r\left(K^{m}\right)=r\left(K^{m+1}\right)=\cdots$. So there exists $0 \neq a_{1} \in K^{m}$ such that $K^{m} a_{1} \neq 0$. Therefore, $K^{2 m} a_{1} \neq 0$. Similarly, there exists $0 \neq a_{2} \in K^{m}$ such that $K^{m} a_{2} a_{1} \neq 0$. Continuous this proceeding, there exists $a_{1}, a_{2}, \cdots \in K^{m}$ such that $a_{n} a_{n-1} \cdots a_{1} \neq 0$, a contradiction. Thus, $K$ is nilpotent.

(3) If there is a sequence $a_{1}, a_{2}, \cdots \in Z_{r}$ such that $a_{n} a_{n-1} \cdots a_{1} \neq 0$ for any positive integer $n$, then $a_{n} a_{n-1} \cdots a_{1} R \neq 0$. Since $r\left(a_{n+1}\right) \leq_{e} R_{R}, r\left(a_{n+1}\right) \cap a_{n} \cdots a_{1} R \neq 0$. This is a contradiction by $(2)$.

Lemma 2.2([8]). Let $a-a c a$ is a regular element for all $a, c \in R$, then $a$ is a regular element.

Lemma 2.3. If $R$ is a right mininjective ring and satisfies $(*)$ such that $S_{r} \leq_{e} R_{R}$, then $R$ is semiprimary.

Proof. Since $R$ is right mininjective, $S_{r} \subseteq S_{l}$. So $J \subseteq l\left(S_{r}\right)$. But $S_{r} \leq_{e} R_{R}$, so 
$l\left(S_{r}\right) \subseteq Z_{r}$. Note that $R$ satisfies $(*)$, then $Z_{r}$ is nilpotent by lemma 2.1. Therefore $Z_{r} \subseteq J$ and so $J=l\left(S_{r}\right)=Z_{r}$ is nilpotent. Next we show that $\bar{R}=R / J$ is regular. If $\bar{a} \neq \overline{0}$, then there exists a nonzero right ideal $K$ such that $r(a) \cap K=0$ by $J=Z_{r}$. Since $S_{r} \leq_{e} R_{R}$, there exists a minimal right ideal $b R \subseteq K$ and So $r(a) \cap b R=0$. Define $f: a b R \rightarrow b R$ by $f(a b r)=b r$ for all $r \in R$. It is clear that $f$ is well-defined. Since $R$ is right mininjective and $a b R$ is a minimal right ideal, there exists $c \in R$ such that $f(a b r)=c a b r$ for all $r \in R$. Thus $b=c a b$ and so $b \in r(a-a c a) \backslash r(a)$. If $a-a c a \in J$, then $\bar{a}$ is a regular element. If not, let $a_{1}=a-a c a$. In the same way we get $a_{2}=a_{1}-a_{1} c_{1} a_{1}$ for some $c_{1} \in R$ and $r\left(a_{1}\right) \subset r\left(a_{2}\right)$. Repeating the above-mentioned process, we get astrictly ascending chain

$$
r\left(a_{1}\right) \subset r\left(a_{2}\right) \subset r\left(a_{3}\right) \subset \cdots,
$$

where $a_{i+1}=a_{i}-a_{i} c_{i} a_{i}$ for some $c_{i} \in R, i=1,2, \cdots$. Let $b_{1}=a_{1}, b_{2}=1-a_{1} c_{1}, b_{3}=$ $1-a_{2} c_{2}, \cdots, b_{i+1}=1-a_{i} c_{i}, \cdots$, then $a_{1}=b_{1}, a_{2}=b_{2} b_{1}, a_{3}=b_{3} b_{2} b_{1}, \cdots, a_{i+1}=$ $b_{i+1} b_{i} \cdots b_{2} b_{1}, \cdots$, whence we have the following strictly ascending chain

$$
r\left(b_{1}\right) \subset r\left(b_{2} b_{1}\right) \subset r\left(b_{3} b_{2} b_{1}\right) \subset \cdots,
$$

which contradicts the hypothesis. So there exists a positive integer $n$ such that $a_{n+1} \in J$, then $\bar{a}$ is a regular element of $\bar{R}$ by lemma 2.2. Therefore $\bar{R}$ is von Neumann regular ring. Finally, we show that $R$ is semilocal. By the proof of $[9$, Theorem 3.4], $\bar{R}$ is semisimple Artinian. Therefore $R$ is semilocal. From above $R$ is semilocal and $J$ is nilpotent. So $R$ is semiprimary.

Theorem 2.1. Let $R$ be a left and right mininjective ring with $J^{2}=0$ and $R$ satisfies $(*)$ in which $S_{r} \leq_{e} R_{R}$. Then $R$ is quasi-Frobenius.

Proof. By Lemma 2.3, $R$ is semiprimary. Thus, $R$ is $Q F$ by [6,Theorem 3.40]

Remark 1. None of the conditions is superfluous in Theorem 2.1. For example, the ring of integers $Z$ is a two-sided mininjective and noetherian ring with $S_{r}=0$. But it is not a $Q F$ ring. Example 2.5 ([6]) indicate that a right mininjective and left artinian ring $R$ is not left mininjective. In fact, $R$ has $A C C$ on both right and left annihilators and so $S_{r}, S_{l}$ are both essential. It is well known that left $P F$ is not right $P F$, and so it is not $Q F$. Indeed, Such ring satisfies the conditions except the chain condition in Theorem 2.1.

Moreover $J^{2}=0$ is needed. For example, If $R$ is a field, the $\operatorname{ring} R=\left(\begin{array}{cc}F & F \\ 0 & F\end{array}\right)$ is a right and left Artinian ring with $J^{2}=0$, but $R$ is neither right or left mininjective. And Example 2.6([6]) indicate that a commutative, local, mininjective ring with $J^{2}=0$ and satisfies $(*)$ such that $S_{r} \leq_{e} R_{R}$ that is not $Q F$.

Corollary 2.1([6, Theorem 3.31]). Let $R$ be a semilocal, left and right mininjective ring with $A C C$ on right annihilators in which $S_{r} \leq_{e} R_{R}$. Then $R$ is quasi-Frobenius. 
Theorem 2.2. The following are equivalent for a ring $R$ :

(1) $R$ is right and left mininjective, right Noetherian, and $S_{r} \leq_{e} R_{R}$.

(2) $R$ is right and left mininjective with $J^{2}=0$, right finitely cogenerated, and $R$ satisfies $(*)$.

(3) $R$ is quasi-Frobenius.

Proof. (1) $\Rightarrow(2)$ and $(3) \Rightarrow(2)$ are clear.

(2) $\Rightarrow(3)$ By lemma 2.1, $Z_{r}$ is right nilpotent. and so $Z_{r} \subseteq J$. Thus $R$ is semilocal and $J=Z_{r}$ by [6, Lemma 8.1]. However, $R$ is semiprimary and so $R$ is $Q F$ by Theorem 2.1.

Since right simple injective ring is right mininjective ring, We can extend Theorem 2.1 to the following theorem:

Theorem 2.3. Let $R$ be a right simple injective ring and satisfies $(*)$ in which $S_{r} \leq_{e} R_{R}$. Then $R$ is quasi-Frobenius.

Proof. By Lemma 2.3, $R$ is semiprimary. Then $R$ is left $P$-injective by [6, Theorem 6.16], which implies that $R$ is left mininjective. Thus $R$ is $Q F$ by Theorem 2.1.

Lemma 2.4. Let $R$ be right $P$-injective and satisfies $(*)$. Then the following hold:

(1) $R$ is right perfect ring.

(2) If $J$ is right T-nilpotent, then $J$ is nilpotent.

(3) $R$ is left perfect ring.

Proof. (1) Assume that for all $a_{i} \in R, i=1,2, \cdots, R a_{1} \supseteq R a_{2} a_{1} \supseteq \ldots$ is descending chain of principal left ideals of $R$. Then $r\left(a_{1}\right) \subseteq r\left(a_{2} a_{1}\right) \subseteq$ ... Since $R$ satisfies $(*)$, then there exists a natraul number $n$ such that $r\left(a_{n} \cdots a_{2} a_{1}\right)=r\left(a_{n+1} a_{n} \cdots a_{2} a_{1}\right)$. Since $R$ is right $P$-injective ring, $R a_{n} \cdots a_{2} a_{1}=$ $l\left(r\left(a_{n} \cdots a_{2} a_{1}\right)\right)=l\left(r\left(a_{n+1} \cdots a_{2} a_{1}\right)\right)=R a_{n+1} \cdots a_{2} a_{1}$. Hence $R$ is right perfect ring .

(2)By hypotheses there exists a natraul number $n$ such that $r\left(J^{n}\right)=r\left(J^{n+1}\right)=\cdots$. Assume $J$ is not nilpotent, then $J^{n+1} \neq 0$ for any $n \in Z^{+}$. So there exists $x_{1} \in J$ such that $J^{n} x_{1} \neq 0$. Therefore, $J^{n+1} x_{1} \neq 0$. Similarly, there exists $x_{2} \in J$ such that $J^{n+1} x_{2} x_{1} \neq 0$. Repeaing the process to obtain $x_{1}, x_{2}, \cdots \in J$ such that $x_{m} \cdots x_{1} \in J \backslash r\left(J^{n}\right)$, a contradiction. Thus, $J$ is nilpotent.

(3) $\mathrm{By}(2), R$ is right perfect ring. Hence, $R / J$ is semisimple and $J$ is right $T$ nilpotent. $\mathrm{By}(3), J$ is nilpotent. So $R$ is left perfect ring.

Theorem 2.4. Let $R$ be a right $P$-injective and satisfies $(*)$ with $J$ is a right $R$ module which have finite Goldie dimension, then $R$ is Artinian ring.

Proof. By lemma 2.4, $R$ is left and right perfect ring, $J$ is nilpotent. So $R / J$ is semisimple Artinian. Let $J^{n}=0, J^{n-1} \neq 0$, then $J^{n-1}$ is semisimple $R / J$-module. By hypotheses, Goldie dimension of $J^{n-1}$ is finite. Hence $J^{n-1}$ is semisimple Artinian $R / J$-module. Since $J \cdot J^{n-2} / J^{n-1}=\overline{0}, J^{n-2} / J^{n-1}$ is semisimple $R / J$-module and Goldie dimension is finite. Thus, $J^{n-2} / J^{n-1}$ is semisimple Artinian. But $R / J$ submodule of $J^{n-1}, J^{n-2} / J^{n-1}$ coincide with $R$-submodule of $J^{n-1}, J^{n-2} / J^{n-1}$. 
Hence $J^{n-1}$ and $J^{n-2} / J^{n-1}$ Artinian $R$-module. Since short sequence of $R$-module

$$
0 \rightarrow J^{n-1} \rightarrow J^{n-2} \rightarrow J^{n-2} / J^{n-1} \rightarrow 0
$$

is exact. Thus $J^{n-2}$ is Artinian $R$-module.

Since $J \cdot J^{n-3} / J^{n-2}=\overline{0}, J^{n-3} / J^{n-2}$ is semisimple $R / J$-module and Goldie dimension is finite. Thus, $J^{n-3} / J^{n-2}$ is $R / J$-module and so is Artinian $R$-module. By short sequence of $R$-module

$$
0 \rightarrow J^{n-2} \rightarrow J^{n-3} \rightarrow J^{n-3} / J^{n-2} \rightarrow 0
$$

is exact. Thus $J^{n-2}$ is Artinian $R$-module. Continuous this proceeding, we get that $J$ is Artinian $R$-module. Therefore, By short sequence of $R$-module

$$
0 \rightarrow J \rightarrow R \rightarrow R / J \rightarrow 0
$$

is exact. Hence $R$ is Artinian ring.

Theorem 2.5. The following are equivalent for a ring $R$ :

(1) $R$ is right simple injective, right Noether ring with $S_{r} \leq_{e} R_{R}$.

(2) $R$ is right simple injective, right finite dimensional, and satisfies (*) with $S_{r} \leq_{e} R_{R}$.

(3) $R$ is quasi-Frobenius.

Proof. (3) $\Rightarrow(1) \Rightarrow(2)$ are clear.

$(2) \Rightarrow(3)$ By lemma $2.3, R$ is semiprimary. Since $R$ is right simple injective, $R$ is right self-injective. Thus, $R$ is Artinian ring by Theorem 2.4. So $R$ is quasiFrobenius.

In general, $R$ is a right $P$-injective and satisfies $(*)$ need not be $\mathrm{QF}$ (see [10]). In the next theorem we show that a right 2-injective and satisfies $(*)$ with $J$ is a right $R$-module which have finite Goldie dimension is quasi-Frobenius.

Theorem 2.6. Let $R$ be a right 2 -injective and satisfies $(*)$ with $J$ is a right $R$ module which have finite Goldie dimension. Then $R$ is quasi-Frobenius.

Proof. By Theorem 2.4, $R$ is left Artinian ring. Then $R$ has $A C C$ on right annihilators. Thus $R$ is $Q F$ by [10, Corollary 3].

Corollary 2.2. $R$ is right $F P$-injective ring satisfies $(*)$ and $J$ is a right $R$-module which have finite Goldie dimension, then $R$ is $Q F$.

A ring $R$ is called left min-CS, if every minimal left ideal is essential in a direct summand of $R_{R}$.

Theorem 2.7. Let $R$ be a left min-CS, left P-injective and satisfies $(*)$ with $J$ is a right $R$-module which have finite Goldie dimension. Then $R$ is quasi-Frobenius.

Proof. By Theorem 2.4, $R$ is right Artinian, then $R$ is a left $G P F$ ring. So $R$ is left Kasch and $S_{r}=S_{l}=S$ by [6, Theorem 5.31], then $S o c(R e)$ is simple for each local idempotent $e \in R$ by [6, Lemma 4.5]. Thus $(e R / e J) \cong l(J) e=S_{r} e=\operatorname{Soc}(R e)$ is 
simple for every local idempotent $e \in R$. Since $R$ is semiperfect, each simple right $R$-module is isomorphic to $e R / e J$ for some local idempotent $e \in R$ by [7, Theorem 27.10]. Hence $R$ is right mininjective by [6, Theorem 2.29]. From above, $R$ is a two-sided mininjective and right Artinian ring, then it is $Q F$ by [6, Theorem 3.31].

Corollary 2.3([11, Theorem 2.21]). If $R$ is right Noetherian left CS and left Pinjective, then $R$ is $Q F$. vspace $0.2 \mathrm{in}$

\section{References}

[1] W. K. Nicholson and M. F. Yousif, Mininjective rings., J. Algebra 187(1997), 548-578.

[2] W. K. Nicholson, J. K. Park and M. F. Yousif, Extensions of simple-injective rings., Comm. Algebra, 28(10)(2000), 4665-4675.

[3] T. Nakayama, On Frobenius Rings. II, Ann. math, 42(1)(1941), 1-21.

[4] C. Faith and D. V. huyhn, When self-injective rings are QF: A report on a problem, Journal of Algebra and Its Application, 1(1)(2002), 75-105.

[5] W. K. Nicholson, and M. F. Yousif, On Quasi-Frobenius Rings, In: Gray F. Birkenmeier, Jae Keol Park and yong Soo Park, International Symposium on Ring Theory, Birkhäuser, Boston. basel. Berlin, 1999.

[6] W.K. Nicholson, and M.F. Yousif, Quasi-Frobenius Rings. Cambridge Tracts in Mathematics 158, Cambridge University Press, 2003.

[7] F. W. Anderson and K. R. Futter, Rings and Modules Categories. New York, SpringerVerlag, 1974.

[8] K. R. Goodearl, Von Neumann regular rings. London, Pitman, 1979.

[9] Chen J. and Ding N., On general principally injective rings., Comm in Algebra, 27(1999), 2097-2116.

[10] E. A. Rutter, Rings with the principal extension property., Comm. Algebra, 3(3)(1975), 203-212.

[11] Chen. J., Li. W., On artiness of right CF rings., Comm. Algebra 32(11),(2004), 44854494. 